\title{
Coordenação dos cuidados em saúde no Brasil: o desafio federal de fortalecer a atenção primária à saúde
}

| ${ }^{1}$ Roberta Marinho da Silva, ${ }^{2}$ Luiz Odorico Monteiro de Andrade I

Resumo: O objetivo do estudo é analisar a indução federal no fortalecimento da atenção primária à saúde (APS) como forma de viabilizar a coordenação dos cuidados no sistema de saúde brasileiro. Estudo quanti-qualitativo, utilizando dados documentais, orçamentários e entrevistas semiestruturadas com gestores federais do Ministério da Saúde (MS) no período de 2007 a 2011. Para o tratamento dos dados foi utilizada análise de conteúdo temática e análise estatística descritiva, que permitiram compreender de forma panorâmica a APS brasileira, especialmente a coordenação dos cuidados, sob a ótica da indução centralizada. O fortalecimento da APS é a base para viabilizar a coordenação dos cuidados no Brasil. Sua priorização vem aumentando no âmbito do MS, o qual vem conduzindo políticas nacionais que impactaram no fortalecimento da APS e na coordenação dos cuidados, tornando-se um forte indutor dessa política, principalmente por meio da edição de portarias. Nenhuma portaria ou orçamento específico foi identificado para a coordenação dos cuidados, o que denota a incipiência desse atributo na APS brasileira. A participação social foi considerada relevante para o alcance da coordenação dos cuidados. A pesquisa conclui que embora a APS tenha sido priorizada na agenda do Ministério da Saúde, sua valorização não ocorreu de forma homogênea em todos os espaços decisórios da esfera federal.

\author{
1 Mestre em Saúde da Família, \\ Universidade Federal do Ceará. \\ Bolsista da Fundação para o \\ Desenvolvimento Científico e \\ Tecnológico em Saúde, FIOTEC, \\ Brasília-DF, Brasil. Endereço \\ eletrônico: robertamarinho88@ \\ gmail.com \\ 2 Professor Adjunto do Curso de \\ Medicina/Sobral, Universidade \\ Federal do Ceará. Fortaleza-CE, \\ Brasil. Endereço eletrônico: \\ odorico0811@gmail.com
}

> Palavras-chave: Atenção primária à saúde; Coordenação dos cuidados em saúde; Ministério da Saúde. 
A coordenação dos cuidados pela atenção primária à saúde (APS) ocupa uma posição de destaque na agenda política de vários países que vêm buscando fortalecer sua atenção primária como uma estratégia de organização do sistema de serviços de saúde.

A segmentação dos sistemas de saúde tem graves consequências para a população, tais como problemas de iniquidade e ineficiência na prestação do cuidado, com duplicidade de funções, desarticulação da rede de atenção, prejudicando o acesso aos níveis de atenção mais complexos, e, portanto, descontinuidade e fragmentação do cuidado (CONILL; FAUSTO, 2007).

Evidências científicas vêm mostrando que os melhores exemplos de sistemas de saúde são os que têm uma APS qualificada e fortalecida, orientadora das açôes e serviços disponíveis nesses sistemas, que oferecem alcance de maior equidade, maior eficiência na continuidade da atenção e satisfação dos usuários (ANDRADE et al., 2012; STARFIELD, 2002).

Hofmarcher et al. (2007) afirmam que medidas pró-coordenação objetivam fortalecer as estruturas da APS. Ao mesmo tempo, são favorecidas por ações e estratégias que tornem a atenção primária mais robusta, mediante o fortalecimento dos seus atributos. Conforme consenso na literatura nacional e internacional, o alcance desses atributos pode ser parâmetro para avaliar a robustez da APS de um determinado sistema de saúde.

Com o status de política federal, a APS brasileira foi gradualmente se tornando central para a reorganização do Sistema Único de Saúde (SUS) e importante para a agenda federal, embora tenha permanecido demasiado tempo "distante das prescrições do SUS” (FACCHINI, 2006).

Sob forte condução federal, a atenção primária passou por uma fase inicial de expansão de cobertura de forma lenta e interiorizada no país. Seguiu para uma mudança de perfil e velocidade com o impulso dado pela execução do Piso de Atenção Básica (PAB), a partir de 1998, e chegou ao Projeto de Expansão e Consolidação da Saúde da Família (PROESF), instituído em 2002 com o objetivo de estimular a expansão da estratégia em grandes centros urbanos. Entre 1998 e 2003, o governo federal aumentou o gasto com APS de 15,68\% para 25,68\% (UGÁ; MARQUES, 2005). Dez anos depois, em 2013, a Estratégia 
Saúde da Família (ESF) alcança a marca de 33.825 equipes, cobrindo 107.065.473 brasileiros, cerca de 55,2\% da população (BRASIL, 2013).

Starfield (2002) defende que a atenção primária possui quatro atributos essenciais, quais sejam: atenção ao primeiro contato, longitudinalidade, integralidade e coordenação. A pesquisadora define esse último atributo como um "estado de estar em harmonia numa ação ou esforço em comum". Sendo assim, a coordenação pode ser definida como a articulação entre diversos serviços e ações de atenção à saúde, de modo que, independentemente de onde sejam prestados, estejam em harmonia e voltados ao alcance de um mesmo objetivo (NÚNEZZ; LORENZO; NAVARRETE, 2006).

Compete à APS realizar essa função, pois ela é a responsável por identificar as necessidades de atendimentos especializados, coordenar as referências para profissionais adequados e garantir a continuidade da atenção acompanhando os resultados terapêuticos e a evolução clínica dos pacientes que percorrem os níveis de atenção no sistema de saúde (ANDRADE; BARRETO; FONSECA, 2006).

\section{Metodologia}

Este artigo apresenta parte dos resultados da pesquisa "Indução centralizada da coordenação dos cuidados pela atenção primária: estudo comparativo entre dois sistemas de saúde universais", realizada entre 2011 e 2013 no Programa de Pós-Graduação em Saúde da Família da Universidade Federal do Ceará, Campus Sobral-CE.

$\mathrm{O}$ estudo tem abordagem mista. A abordagem qualitativa, principal núcleo reflexivo, trabalhou os instrumentos normativos e o discurso dos principais sujeitos que atuaram na APS no âmbito do Ministério da Saúde durante os anos 2007 a 2011. A abordagem quantitativa visou sistematizar os dados documentais e apontar elementos de base financeira que caracterizaram a indução federal da atenção primária.

O recorte temporal, de 2007 a 2011, corresponde ao período em que vigorou o Pacto pela Saúde, regulamentado pela Portaria GM/MS No 699/2006. O Pacto trouxe "a marca da maturidade" para a APS ao definir como prioridades a consolidação e a qualificação da Estratégia Saúde da Família como modelo de APS e centro ordenador das redes de atenção à saúde no SUS. O pacto tem seu 
desfecho com o lançamento do Decreto No 7.508/2011, que demarca outra fase na gestão do sistema de saúde brasileiro.

Identificamos as portarias expedidas no período de janeiro de 2007 a dezembro de 2011 por meio de consulta sistemática à base de dados do Sistema de Legislação da Saúde do Ministério da Saúde - Saúde Legis, no site http://portal2.saude.gov. br/saudelegis. Foram utilizadas as seguintes palavras-chave: Atenção Básica; Atenção Primária; Saúde da Família; Núcleos de Apoio à Saúde da Família (NASF); Projeto de Expansão e Consolidação da Saúde da Família (PROESF); Agentes Comunitários de Saúde (ACS); PAB fixo e PAB variável; e Programa Nacional de Melhoria do Acesso e da Qualidade da Atenção Básica (PMAQ).

Os sujeitos entrevistados foram os Diretores do Departamento de Atenção Básica (DAB) da Secretaria de Assistência à Saúde (SAS) do Ministério da Saúde e o Coordenador Geral da Gerência em Atenção Básica do DAB/SAS. Todos atuaram no período de 2007 a 2011.

Quanto aos dados orçamentários, trabalhamos principalmente os Blocos de APS e da Atenção de Média e Alta Complexidade Ambulatorial e Hospitalar (MAC) referentes aos cinco anos em estudo. A fonte utilizada foi a base de dados de recursos federais do SUS, gerenciada pelo DATASUS, tendo sido identificados no site do Portal da Saúde, do DATASUS, na Sala de Apoio à Gestão Estratégica (Gestão e Financiamento, Transferência Fundo a Fundo, Competência).

A pesquisa foi aprovada pelo Comitê de Ética em Pesquisa da Universidade Estadual Vale do Acaraú (Parecer no 198.092), sendo considerada uma pesquisa relevante no cenário da Saúde Coletiva brasileira.

\section{Resultados e discussão}

A atenção primária brasileira foi estruturada em meio ao intenso processo de descentralização das políticas sociais e de municipalização do poder federativo. Admitiram-se múltiplos partícipes do SUS, fruto de acordos políticos e do desenvolvimento de instrumentos técnico-operacionais necessários à integração e à manutenção da unicidade do sistema, mediante reformulação dos papéis e das funções dos entes governamentais na oferta de serviços, na gerência de unidades e na gestão do sistema de saúde (VIANA et al., 2002). 
Os gestores federais consideram que a estruturação da APS brasileira se deu de forma tardia, com início nos anos 1990 e levando mais uma década para sua estruturação como política pública, além de só recentemente ter ultrapassado uma cobertura populacional superior a 50\%. Esse fato interferiu diretamente no seu papel de coordenadora do sistema, uma vez que a cobertura universal é fundamental para o atendimento desse atributo.

Nesse sentido, destaca-se a inclusão da ESF no Programa de Aceleração do Crescimento (PAC), responsável pela garantia de maior acesso e cobertura à APS, com o Programa de Requalificação das Unidades Básicas de Saúde (UBS). Em parceria com os estados e municípios, o governo federal vem investindo em modernização e qualificação do atendimento à saúde para a população, viabilizando a construção de mais de três mil novas UBS e reformando, ampliando e informatizando mais de 20 mil unidades (BRASIL, 2012).

O desfecho da utilização dos recursos do PAC na atenção primária ocorreu com a publicação da Portaria $n^{\circ} 837$, de 23 de abril de 2009, que integra o bloco de investimentos aos blocos de financiamento relativos à transferência de recursos federais para as ações e os serviços de saúde no âmbito do SUS. Com a instituição desse bloco, foi regulamentado o Plano Nacional de Implantação de Unidades Básicas de Saúde para Equipes de Saúde da Família: o Programa de Requalificação de UBS, em 2009, e o respectivo Componente Reforma, em 2011, que posteriormente foi ampliado e, em 2013, redefinido também mediante portaria. Esses incentivos foram citados pelos gestores como importante passo para a atenção primária brasileira.

Segundo Castro (2009), o Ministério da Saúde fez do Programa Saúde da Família (PSF) uma política governamental prioritária no período de 1998 e 2002, representando a estratégia escolhida para expandir e reorganizar a APS no país. A autora concluiu que, no período de 2003 a 2008, "a atenção primária à saúde ocupou lugar de destaque na agenda de prioridades do Ministério da Saúde no governo Lula”. Essa prioridade anunciada pelo Ministro da Saúde em 2003 foi seguida de maiores investimentos na atenção primária e no PSF, tendo o ministro afirmado que a perspectiva para o programa seria de maior flexibilidade para atender diferentes realidades locais. 
Os gestores federais da atenção primária mantiveram acordo em afirmar que o DAB direcionou esforços para o desenvolvimento da APS buscando a ampliação do PSF para todo o Brasil, bem como maiores fontes de financiamento para a atenção primária. Suscitou-se que a APS foi priorizada na gestão dos dois ministros da saúde anteriores, José Agenor Álvares da Silva (2006 e 2007) e José Gomes Temporão (2007 a 2010), o que se intensificou na gestão do Ministro Alexandre Padilha (2011 a 2014), com incremento dos investimentos na atenção primária e com maior coesão interna no governo.

Podemos concluir que cada momento de construção da APS brasileira, assim como é inerente ao processo de desenvolvimento de toda a política de saúde, configura-se como "um processo composto de uma sequência de tomada de decisões com relação a como enfrentar um problema identificado como prioritário na agenda pública”. Sendo o SUS um espaço marcado por amplas arenas decisórias e instâncias de pactuação, podemos afirmar que a APS também envolve etapas de elaboração, implantação e execução, das quais participam diversos atores, que compõem um círculo de relações de poder, expressos em espaços específicos denominados "arenas", e que moldam o formato geral dessa política (FLEURY; OUVERNEY, 2008).

A política de saúde pode ser compreendida como uma "resposta (ação ou omissão) de uma organização (como o Estado) diante das condições de saúde dos indivíduos e das populações” e dos determinantes e relações que afetam a saúde humana e o ambiente (PAIM; TEIXEIRA, 2006). É preciso considerar também que as políticas de saúde abrangem questóes referentes ao poder e ao estabelecimento de diretrizes, planos e programas de saúde, além de compreenderem as relações de poder na implantação de políticas em geral (FLEURY; OUVERNEY, 2008).

A APS, como política de saúde, é significativamente sensível às forças de coalizão típicas do nosso sistema presidencialista e federalista. Ademais, a construção da ESF, modelo estruturante da APS brasileira, foi consequência de um processo lento e contínuo de tensão com o modelo hegemônico de assistência à saúde, uma vez que visava superar a incapacidade do modelo hegemônico tecnicista, hospitalocêntrico e medicalocêntrico na intenção de responder efetivamente às demandas individuais e coletivas (ANDRADE et al., 2012). A 
ESF é apontada por um dos gestores, portanto, como contra-hegemônica no cenário da política de saúde.

Um dos sujeitos afirmou ainda que um dos principais objetivos do Departamento de Atenção Básica era garantir que o modelo brasileiro de APS escolhido fosse de fato a Estratégia Saúde da Família, preocupação existente mesmo após a publicação da Política Nacional de Atenção Básica (PNAB) em 2006.

Quanto à defesa da APS nas instâncias de pactuação, decisão e execução da política de saúde, seja no nível de governo, ministério, secretaria, departamento ou mesmo de um esforço pessoal, os gestores federais apresentaram diferentes opiniōes acerca da priorização da APS na agenda política federal. As opiniōes passaram pela afirmação de que a APS é contra-hegemônica até mesmo na esfera federal; pelo reconhecimento de que a APS foi fortemente defendida nos discursos no âmbito do Ministério da Saúde, mas que a defesa pela melhoria no financiamento se restringiu ao DAB; até opiniōes que expressaram claramente o período como sendo de grande priorização da APS.

Internamente ao Ministério da Saúde, foi citado pelos gestores o apoio ao DAB por parte da Secretaria de Vigilância à Saúde (SVS), da Secretaria Executiva (SE) e da Secretaria de Gestão do Trabalho e da Educação na Saúde (SGTES). Em relação à parceria dos Conselhos de Saúde, houve discordância quanto ao apoio na defesa pela APS. Foi confirmado o apoio dos conselhos como parceiros nas discussões da Comissão Intergestores Tripartite, mas também posturas de inconstância, por vezes fazendo críticas à ESF, e não uma defesa intransigente da política de atenção básica.

O Conselho Nacional Secretários de Saúde (CONASS) e o de Secretarias Municipais de Saúde (CONASEMS) foram citados como grandes parceiros, influenciando inclusive o lançamento da PNAB, se considerarmos que em um tempo relativamente curto o Ministério da Saúde conseguiu revogar cerca de 40 portarias, provenientes de emendas feitas à Portaria MS/GM N.o 1.886/1996, que trouxe as normas de funcionamento do PSF e do Programa de Agentes Comunitários de Saúde. Na opinião do gestor, esse feito demarca uma priorização da APS por parte do Ministério da Saúde, pelo fato de diminuir a grande fragmentação normativa que prevalecia até o lançamento da PNAB em 2006, em que havia sucessivamente a edição de emendas que, ao buscarem adequação 
às novas realidades apresentadas e comporem o processo de implementação da APS no Brasil, nem sempre seguiam uma única lógica, tal como propôs a PNAB, com seus princípios e diretrizes.

Os gestores citaram ainda que a participação social tem relevância significativa na condução de políticas públicas, em especial por meio dos Conselhos de Saúde, que têm na APS um locus privilegiado de atuação no sistema de saúde, mas que ainda são pouco divulgados à comunidade. Segundo Labra (2005), esta muitas vezes desconhece seus objetivos, funções e formas de atuação, resultando em baixa adesão nos processos decisórios locais. A baixa divulgação da ESF, tal como acontece com os conselhos, também é um fator que enfraquece a atuação social no SUS, configurando-se, por conseguinte, como importante barreira para o fortalecimento da APS no Brasil, que, pelo seu contingente populacional, deveria contar com significativa força popular.

Observando aspectos das políticas nacionais que contribuíram para a coordenação dos cuidados pela APS de 2007 a 2011, nenhuma apresentou como objetivo específico alcançar a coordenação dos cuidados pela APS. Entretanto, sobressaíram com potencial para impactar sobre o fortalecimento da APS e, consequentemente, sobre a coordenação do cuidado, a PNAB, os NASF, as Redes de Atenção à Saúde (RAS) e o PMAQ.

A PNAB de 2006 foi considerada um marco normativo, destacando-se pela qualidade de sua construção do ponto de vista ideológico e político, ao definir responsabilidades para os entes federativos, garantindo a participação da União e incluindo as conquistas da ESF desde 1994, englobando até mesmo o que nem estava publicado em portaria. Atribuiu-se à PNAB a garantia da ESF como principal modelo de APS brasileiro, alcançando um status de modelo de atenção primária para outros países, e ainda com a vantagem de ter equipes multidisciplinares em alguns lugares e um quantitativo de equipes NASF em ascensão.

Como desenvolvimento da PNAB, os Cadernos de Atenção Básica, da Série Pactos pela Saúde (que funcionam como protocolos e parâmetros técnicos desenvolvidos pelo Ministério da Saúde), foram compreendidos como bastante pertinentes e úteis à coordenação dos cuidados, por qualificar os processos de referência e contrarreferência entre os pontos de atenção. 
Já a PNAB 2011 surgiu sem grandes mudanças, porém, com maior ênfase nos conceitos de rede e de linhas de cuidado a partir do Decreto no 7.508/2011. Além disso, permitiu que o Ministério da Saúde incluísse outros modelos de APS, atendendo à demanda de alguns municípios que construíram seus modelos de APS diferentes da ESF, adotada pelo governo federal.

A criação do NASF foi um dos desdobramentos da PNAB 2011, que já vinha se concretizando desde 2008. O NASF foi ressaltado como importante estratégia para aumentar a resolubilidade da APS e para qualificar os encaminhamentos entre a ESF e os demais níveis de atenção, sendo considerado até mesmo a principal estratégia de atuação junto à ESF na coordenação dos cuidados.

O lançamento das RAS foi citado como fato muito importante no contexto organizacional do SUS, mas ainda insuficiente para a coordenação dos cuidados pela APS. Ressaltaram que a política de RAS, no formato em que foi lançada, apresentou-se como evidência negativa para o atributo em questão, pois foi desenhada para atender a um segmento específico de usuários e se desdobrou em portarias temáticas que atomizaram a lógica de Rede de Atenção à Saúde compreendida no âmbito das necessidades dos usuários e da capacidade instalada de uma região. Os gestores destacaram-na como ponto inicial do sistema público de saúde e com possiblidades de evolução. Contudo, ela deve ser acompanhada de outras estratégias e mecanismos concretos de apoio à organização logística, além de serem inadiavelmente necessários maiores investimentos estruturais na APS.

O PMAQ foi citado como exemplo de estratégia com potencial para impulsionar a coordenação do cuidado pela APS. Esse programa é composto por quatro fases distintas: adesão e contratualização, desenvolvimento, avaliação externa e recontratualização. A terceira fase equivale ao espaço de participação social, com avaliação da satisfação do usuário, junto à avaliação da rede local de saúde pelas equipes da atenção básica e ao estudo de base populacional sobre aspectos do acesso, utilização e qualidade da atenção básica (BRASIL, 2011). Segundo Felisberto et al. (2010), o PMAQ também apresenta potencial para qualificação dos processos decisórios, no âmbito da gestão dos serviços e do cuidado, tendo como finalidade a integralidade e resolubilidade das ações, considerada a incipiência das práticas de monitoramento e avaliação nas três esferas de gestão. 
É essencial discutir os impactos das macropolíticas na APS e na coordenação dos cuidados para compreender esse processo sob a gestão federal. Com a divisão de responsabilidades para execução das políticas públicas, a União tornou-se a maior responsável pela formulação das políticas, arrecadação fiscal e devolução de recursos, na forma de transferências obrigatórias e voluntárias. Já os estados orientaram-se ao papel de "alavancas do desenvolvimento", ao passo que os municípios ficaram relegados a segundo plano no que diz respeito a seu envolvimento com as políticas sociais, tornando-se, no entanto, gestores fundamentais das políticas em sua operacionalidade (LASSANCE, 2012).

Essas inferências permitem compreender de forma panorâmica o momento em que se encontra a APS brasileira e, em especial, a coordenação dos cuidados, sob a ótica da indução centralizada no Brasil. Reconhecemos a limitação deste estudo quanto ao escopo adotado, que não abrange os demais entes federados na condução desse processo interfederativo. Ressaltamos a importância de pesquisas que ampliem a compreensão do atributo coordenação dos cuidados também em outras esferas de gestão do SUS, com vistas à formulação de políticas com impactos efetivos na APS.

\section{Condução ministerial da atenção primária à saúde mediante expedição de portarias nos anos 2007 a 2011}

Nas instâncias normativas, mesmo após a publicação da PNAB, consideramos que o principal instrumento de coordenação das ações nacionais de saúde, principalmente a partir da década de 1990, tem sido a edição de portarias ministeriais. Elas têm grande relevância no contexto da saúde, não só por sua vultosa quantidade, superior a mil portarias ao ano, mas, principalmente por sua forte capacidade de indução adquirida na definição da política setorial, expressando-se como uma forma de condução da política de saúde, com predomínio da esfera federal (BAPTISTA, 2007).

Portanto, reforçamos a necessidade de buscar todas as portarias pertinentes ao estudo e, após eliminação de duplicidades, obtivemos um total de 696 portarias assinadas e mais quatro normativas de duas modalidades menos utilizadas, a portaria conjunta e a consulta pública, para o período de janeiro de 2007 a dezembro de 2011 (Quadro 1). 
Quadro 1. Normativas assinadas no âmbito federal acerca da atenção primária à saúde, 2007-2011

\begin{tabular}{|c|c|c|c|c|c|}
\hline ANO & NORMA & QUANTIDADE & REVOGADAS & VIGENTES & ORIGEM \\
\hline 2007 & $\begin{array}{l}1 \mathrm{COP} \\
1 \mathrm{POC} \\
88 \text { PRT }\end{array}$ & 90 & 6 PRT & $\begin{array}{c}84 \\
\text { NORMAS }\end{array}$ & $\begin{array}{l}67 \mathrm{GM}, 13 \mathrm{SAS} \\
9 \mathrm{SE}, 1 \mathrm{SAS} / \mathrm{SE}\end{array}$ \\
\hline 2008 & 117 PRT & 117 & 8 PRT & 109 PRT & $\begin{array}{l}73 \text { GM, } 21 \text { SE } \\
19 \text { SAS, } 3 \text { SE/ } \\
\text { FNS } \\
1 \text { SVS }\end{array}$ \\
\hline 2009 & 187 PRT & 187 & 2 PRT & 185 PRT & $\begin{array}{l}150 \text { GM, } \\
25 \text { SE/FNS } \\
8 \text { SAS, } 3 \text { SE } \\
\quad 1 \text { SVS }\end{array}$ \\
\hline 2010 & $\begin{array}{c}1 \text { POC } \\
140 \text { PRT }\end{array}$ & 141 & 8 PRT & $\begin{array}{c}133 \\
\text { NORMAS }\end{array}$ & $\begin{array}{l}104 \text { GM, } \\
20 \text { SE/FNS } \\
13 \text { SAS, } 3 \text { SVS } \\
1 \text { SGTES }\end{array}$ \\
\hline 2011 & $\begin{array}{l}1 \text { POC } \\
164 \text { PRT }\end{array}$ & 165 & 3 PRT & $\begin{array}{c}162 \\
\text { NORMAS }\end{array}$ & $\begin{array}{l}151 \text { GM, } 11 \\
\text { SAS } \\
2 \text { CNS, } 1 \text { SE/ } \\
\text { FNS }\end{array}$ \\
\hline TOTAL & $\begin{array}{c}1 \text { COP } \\
3 \text { POC } \\
696 \text { PRT }\end{array}$ & 700 & 27 & 673 & $\begin{array}{c}545 \text { GM, } 64 \\
\text { SAS } \\
49 \text { SE/FNS } \\
33 \text { SE, } 5 \text { SVS } \\
2 \text { CNS, } 1 \text { SAS/ } \\
\text { SE } \\
1 \text { SGTES }\end{array}$ \\
\hline LEGENDA & \multicolumn{5}{|c|}{$\begin{array}{l}\text { POC (Portaria Conjunta); COP (Consulta Pública); PRT (Portaria); } \\
\text { GM (Gabinete do Ministro); SAS (Secretaria de Atenção à Saúde); SE (Secretaria } \\
\text { Executiva) / FNS (Fundo Nacional de Saúde); SVS (Secretaria de Vigilância à } \\
\text { Saúde); CNS (Conselho Nacional de Saúde); SGTES (Secretaria de Gestão de } \\
\text { Gestão do Trabalho e da Educação na Saúde). }\end{array}$} \\
\hline
\end{tabular}

Fonte: Elaboração própria. 
O estudo realizado por Castro (2009) também identificou numerosas portarias sobre APS, expedidas entre 2003 e 2008, num total de 608. A autora ressalta que na década de 1990 ampliou-se o poder do Ministério da Saúde quanto à indução e a regulação sobre estados e municípios mediante a edição de normas e portarias atreladas a mecanismos financeiros. Ela conclui que houve uma redução no papel de execução direta das ações e serviços de saúde pelo Ministério da Saúde e maior ênfase na regulação e na sua função de financiador de políticas e programas.

Neste estudo, destacamos a vultosa quantidade de normativas e a esmagadora maioria do formato em portarias (Quadro 1). Também refletimos sobre a origem da edição desses instrumentos na condução da política de saúde: só o gabinete do ministro editou $77,86 \%$ das normativas ( $\mathrm{n}=545)$. Outra questão levantada é acerca do seu conteúdo, uma vez que todas guardam relação direta ou indireta com a APS brasileira nos anos de vigência do Pacto pela Saúde.

Assim, elaboramos o Quadro 2, sistematizando o conteúdo destas normativas em cinco eixos principais: i) Incentivos, Habilitações, Credenciamentos e Autorizações diretas para incentivos financeiros; ii) Operacionalização de planos, políticas e programas; iii) Suspensão direta de incentivos financeiros, por irregularidade em cadastro ou por avaliação da Secretaria Estadual de Saúde (SES), do Departamento Nacional de Auditoria do SUS (DENASUS) ou dos órgãos de controle externos ao SUS; iv) (Re)definição, Estabelecimento, Instituição, Regulamentação, Alteração e Autorização de recursos financeiros; v) Alterações de portarias anteriores e designações de cargos no DAB/SAS.

Quadro 2. Conteúdo das normativas assinadas no âmbito federal acerca da atenção primária à saúde, 2007-2011

\begin{tabular}{|l|c|c|c|c|c|c|}
\hline CONTEÚDO DA NORMA & $\mathbf{2 0 0 7}$ & $\mathbf{2 0 0 8}$ & $\mathbf{2 0 0 9}$ & $\mathbf{2 0 1 0}$ & $\mathbf{2 0 1 1}$ & TOTAL \\
\hline $\begin{array}{l}\text { i) Incentivos, Habilitaçóes, } \\
\text { Credenciamentos e Autorizaçóes diretas } \\
\text { para incentivos financeiros }\end{array}$ & 37 & 46 & 88 & 47 & 59 & $\mathbf{2 7 7}$ \\
\hline $\begin{array}{l}\text { ii) Estritamente sobre } \\
\text { operacionalizaçáo de planos, políticas e } \\
\text { programas }\end{array}$ & 26 & 35 & 47 & 45 & 28 & $\mathbf{1 8 1}$ \\
\hline
\end{tabular}




\begin{tabular}{|l|c|c|c|c|c|c|}
\hline CONTEÚDO DA NORMA & $\mathbf{2 0 0 7}$ & $\mathbf{2 0 0 8}$ & $\mathbf{2 0 0 9}$ & $\mathbf{2 0 1 0}$ & $\mathbf{2 0 1 1}$ & TOTAL \\
\hline $\begin{array}{l}\text { iii) Suspensão direta de incentivos } \\
\text { financeiros, por irregularidade em } \\
\text { cadastro ou por avaliação da SES ou do } \\
\text { DENASUS ou dos órgãos de controle } \\
\text { externos ao SUS }\end{array}$ & 18 & 20 & 32 & 23 & 59 & $\mathbf{1 5 2}$ \\
\hline $\begin{array}{l}\text { iv) (Re)definição, Estabelecimento, } \\
\text { Instituição, Regulamentaçáo, Alteração } \\
\text { e Autorizaçáo de recursos financeiros }\end{array}$ & 7 & 13 & 18 & 15 & 17 & $\mathbf{7 0}$ \\
\hline $\begin{array}{l}\text { v) Alterações de portarias anteriores e } \\
\text { designaçóes de cargos no DAB/SAS }\end{array}$ & 2 & 3 & 2 & 11 & 2 & $\mathbf{2 0}$ \\
\hline TOTAL & $\mathbf{9 0}$ & $\mathbf{1 1 7}$ & $\mathbf{1 8 7}$ & $\mathbf{1 4 1}$ & $\mathbf{1 6 5}$ & 700 \\
\hline
\end{tabular}

Fonte: Elaboração própria.

Concluímos que a maioria das normativas se concentra no primeiro eixo, "Incentivos, Habilitações, Credenciamentos e Autorizações diretas para incentivos financeiros", representando 39,57\% das 700 normativas sobre APS para o período estudado. Além disso, o terceiro eixo, referente a suspensões financeiras no âmbito da APS, representou $21,71 \%$ das normativas para o período. Ambos os eixos somados resultaram na maioria das normativas lançadas pelo Ministério da Saúde (61,28\%), sem contar que o quarto eixo (com 10\%) também se refere a mecanismos condicionadores de recursos financeiros, embora de forma indireta.

Esses dados revelam que o Ministério da Saúde adentrou pela terceira década (ano de 2011) imbuído dos papeís de importante financiador das políticas e programas no que diz respeito à APS e, portanto, de forte indutor do desenvolvimento da APS brasileira.

Quanto à evolução das normativas, percebemos constância em sua quantidade durante todo o período, mas sublinhamos o terceiro eixo, acerca das suspensões financeiras no âmbito da APS, em que houve uma mudança quantitativa de uma média de 23 normativas de 2007 a 2010, para 59 em 2011. Esse dado pode mostrar uma maior fiscalização pelo Ministério da Saúde, pelas Secretarias Estaduais de Saúde e pelos órgãos de controle externos, mas também um aumento do número 
de irregularidades na implementação da PNAB ou mesmo no preenchimento dos sistemas de informação, junto a um maior rigor na análise das informaçôes contidas nas bases de dados do Ministério da Saúde.

A partir do conteúdo expresso nas portarias classificadas no terceiro eixo, identificamos os principais motivos para suspensão direta de incentivos financeiros na APS. No que tange às bases de dados SCNES e SIAB, a principal causa era referente ao número de equipes de saúde da família, equipes de saúde bucal e de agentes comunitários de saúde nos municípios com irregularidade no cadastro de profissionais. No caso do Ministério da Saúde (área técnica), a suspensão tinha como causa a desativação de equipe de saúde família ou de saúde bucal no município, havendo suspensão também de equipe NASF por não ter alcançado metas físico-financeiras do estado. Quanto à SES, DENASUS, CGU, TCU e MP, as questóes ficaram circunscritas à irregularidade na carga horária das equipes (geralmente do profissional médico, mas também do odontólogo e raramente do enfermeiro, apenas uma por ausência deste) ou por composição incompleta das equipes, sendo que em 2011, todos esses motivos recaíram sobre a parte variável do Piso de Atenção Básica.

De antemão, reconhecemos que algumas irregularidades identificadas como simples falhas nos cadastros podem não ser apenas um problema ou desatualização no registro de informações, mas sim um problema da própria realidade local, com equipes em funcionamento irregular, como, por exemplo, a falta de algum profissional que deveria compor a equipe mínima, mesmo temporariamente.

Baptista (2007) destaca que "a discussão das normas possibilitou na última década um amadurecimento dos gestores dos três níveis de governo sobre os problemas e desafios a serem enfrentados na construção do SUS”. Ressalta ser um entrave a edição de muitas portarias, pois nesse processo criam-se novas regras que alteram o que havia sido estabelecido após ampla discussão e pactuação, sem uma nova rodada de discussão com os segmentos interessados, situação que prejudica o processo decisório no SUS.

A partir da análise das 700 normativas, concluímos que nenhuma portaria referente ao período estudado teve como objeto principal a coordenação dos cuidados pela APS, o que denota a incipiência desse atributo nas políticas de 
saúde brasileiras, fato preocupante, se for considerada a relevância dessa questão para a qualidade do sistema como um todo.

\section{Financiamento federal da atenção primária brasileira e o desafio de estimular a coordenação dos cuidados}

O financiamento federal da atenção primária chegou à fase do Pacto pela Saúde, em 2006, após um período de quase dez anos de amadurecimento, se consideramos que esse financiamento se configurou de forma sistemática a partir de 1998, quando foi instituído o PAB e se engendrou a efetiva descentralização das ações da APS brasileira.

A implantação do PAB consolidou o repasse federal feito diretamente aos municípios, mediante transferência regular e automática com base em um valor per capita repassado de forma direta aos Fundos de Saúde dos municípios habilitados. Castro e Machado (2010) destacam que embora houvesse um esforço para reajustar importantes incentivos, o PAB fixo ficou sem alteração desde a sua implantação até 2003. Segundo Solla et al. (2007), a partir de 2003, o Ministério da Saúde estabeleceu uma estratégia de correção progressiva do PAB fixo, que teve seu valor mínimo aumentado, saindo de $\mathrm{R} \$ 10,00$ para $\mathrm{R} \$ 13,00$, estabelecendo meta de $\mathrm{R} \$ 18,00$ no segundo semestre de 2006. Ademais, a correção da base populacional para cálculo do PAB fixo passou a ser feita anualmente. Tudo isso pode ser considerado como priorização da APS pela esfera federal.

Já em 2006, o financiamento do SUS teve como pano de fundo o Pacto pela Saúde, que respaldou financeiramente a PNAB de 2006. A partir de então, o PAB fixo e o PAB variável passaram a compor o Bloco de Atenção Básica.

Durante os anos de 2007 e 2011, o valor repassado pelo Bloco de Atenção Básica teve um crescimento significativo, como se pode observar no Gráfico 1, onde constam os valores (em reais) repassados do Fundo Nacional de Saúde para os Fundos de Saúde dos estados, do Distrito Federal e dos municípios. Ainda neste gráfico, dispomos também os valores referentes aos recursos de MAC, que historicamente recebem as maiores parcelas do orçamento do SUS. Neste gráfico, podemos visualizar a ênfase no distanciamento entre as curvas que representam os valores repassados para os Blocos de Atenção Básica e de MAC, tal como acontecia antes da criação dos blocos de financiamento. 


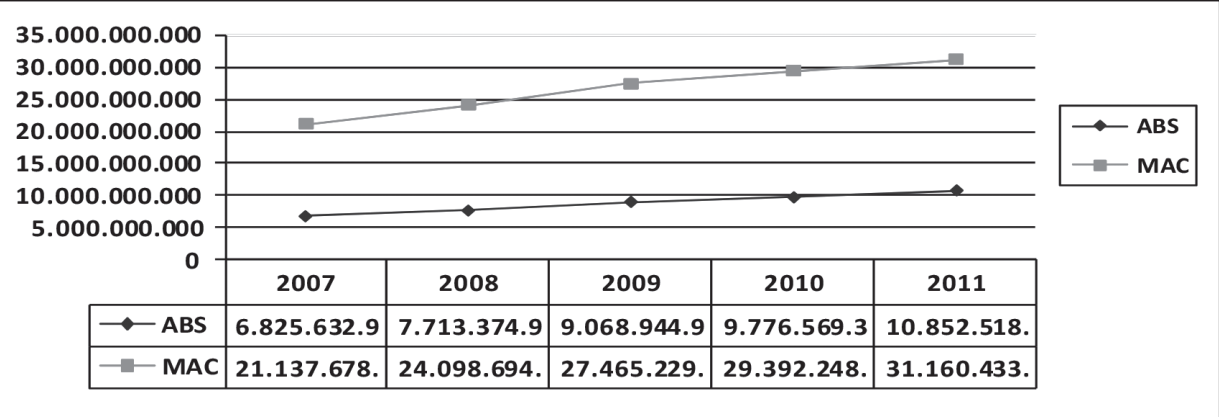

Fonte: Sala de Apoio à Gestão Estratégica, Gestão e Financiamento, do DATASUS, Ministério da Saúde.

Apesar de permanecer a discrepância entre os valores repassados para o Bloco de Atenção Básica e o de MAC, notamos que o aumento percentual de recursos para cada um desses blocos manteve-se semelhante, à exceção de 2011, ano em que houve um aumento de 9,91\% nos recursos de MAC em relação a 2010, ao passo que o Bloco de ABS aumentou apenas 5,67\%, representando o menor aumento percentual desde 2007.

Esse dado contrasta, em parte, com o resultado encontrado por Castro e Machado (2010) sobre o período de 1998 a 2006, em que a ABS manteve um padrão de progressivo incremento entre 1998 e 2001 e de 2003 a 2006, enquanto as transferências de recursos para MAC apresentaram oscilações ao longo dos anos, com valores per capita muito superiores em todo o período, e crescimento bem ascendente entre 2002 e 2004, acentuando a distância entre o financiamento de ABS e de MAC.

Concluímos que, para o financiamento entre os anos 2007 e 2011, os recursos de ABS e de MAC tiveram progressivo padrão de incremento nos cinco anos do estudo, embora esse incremento tenha diminuído para quase metade nos últimos dois anos, no caso da atenção básica, porém de forma mais acentuada no caso da MAC. Essa pequena vantagem da APS em relação aos recursos MAC não impediu, todavia, que as curvas entre os recursos de $\mathrm{ABS}$ e de MAC se mantivessem distantes em todo o período. 
Essa diferença não se restringe apenas aos dois blocos analisados, $\mathrm{ABS}$ e MAC, haja vista que o financiamento repassado para os demais blocos também é bastante inferior em comparação ao valor transferido para o Bloco de MAC. Sobre essa nítida diferença, justificada principalmente por ocorrer nas complexidades média e alta, onde funcionam as tecnologias duras e, portanto, os procedimentos mais onerosos para o sistema de saúde, os gestores criticaram a extrema priorização dos recursos para MAC em detrimento da APS e enfatizaram a forma como os recursos de MAC são geridos, bem como a sua insuficiente oferta, pois estão entre os principais desafios a serem trabalhados na busca de alcançar a coordenação dos cuidados pela APS (ALMEIDA, 2010).

A gestão do financiamento da APS tem um modelo estruturado com base populacional e territorial, o que não ocorre com o financiamento da atenção especializada, a qual permanece sendo orientada por produção de serviços tabelados pelo SUS.

Desde a criação do PAB, o financiamento da APS deixou de utilizar a tabela de procedimentos do SUS, passando a utilizar valores per capita, embora tenha incluído procedimentos dessa tabela, construída com participação dos três entes federados sob condução federal. Logo, o "DNA" ambulatorial e hospitalar permaneceu enraizado, e, com ele, a lógica de pensar e compreender a estrutura financeira do SUS, cujo financiamento ainda se configura em sua grande parte estratificado por procedimentos e atendendo a essa tabela, o que nos faz levantar a hipótese de haver também aqui um importante gargalo para a coordenação dos cuidados no interior do sistema de saúde brasileiro.

Sobre a coordenação dos cuidados pela APS, um dos gestores afirmou que "não dá pra dizer que tem um incentivo específico para fortalecer a coordenação do cuidado" e ponderou sobre o que poderia ser feito nesse sentido, como atrelar apoio diagnóstico e terapêutico à PNAB, de forma que impulsionasse financeiramente o funcionamento de dispositivos de coordenação dos cuidados no âmbito da APS. Para a possibilidade concreta dessa sugestão, seria necessário aumentar o financiamento do Bloco de Atenção Básica, além de repensar a estrutura da APS, mas, principalmente fazer essas alteraçôes sem ferir seus princípios e diretrizes. 
$\mathrm{Na}$ intenção de aumentar os recursos de APS, foi citada a possibilidade de criação da Secretaria de Atenção Básica dentro do Ministério da Saúde, em substituição à atual estrutura de departamento, que concorre a financiamento com os demais departamentos de sua secretaria. Uma vez que o DAB passasse a ser uma secretaria, estaria ligado diretamente ao gabinete do ministro, tendo, portanto, ampliadas as chances de a APS ter maior apreciação em suas ações e prioridades e, consequentemente, alcançar um aumento quantitativo no seu financiamento.

O gestor acredita que a proposta de separação das secretarias e, por conseguinte, do financiamento seria algo positivo para a APS e não recairia em maior fragmentação devido ao financiamento da APS utilizar recurso per capita, diferente dos demais recursos da política assistencial, que ocorrem por tabela de produção. Como isso não aconteceu, o gestor destacou a "impossibilidade de avaliar como seria a separação dos recursos da APS da Atenção Especializada porque a criação da Secretaria de APS não aconteceu", mas defendeu seu ponto de vista argumentando que os esforços para aumentar o financiamento da APS sofrem com os reveses de lutar por incentivos per capita, ficando em desvantagem em comparação aos incentivos ofertados à tabela de procedimentos do SUS.

Entre os problemas ocasionados com o privilégio dos recursos para MAC, destaca-se a tendência à iniquidade financeira atrelada a esse mecanismo, que favorece regiôes do país economicamente mais desenvolvidas. Mesmo com a Compensação de Especificidades Regionais mediante o PAB variável e com o emprego, a partir de 2004, de "indicadores de condições sociais como critério para diferenciar os repasses federais aos municípios" (SOLLA et al., 2007), ainda se faz necessário o combate às marcantes desigualdades regionais, o que implica em "maior aporte de recursos e transformações mais substantivas no financiamento federal da saúde" (CASTRO; MACHADO, 2010).

Isso nos faz concluir que, neste momento, mais do que nunca, precisamos resgatar as "promessas" para o SUS. A busca da vinculação de recursos para o setor saúde é uma situação que vem sendo tensionada por meio das muitas propostas de emenda constitucional construídas na década de 1990 (OCKÉ-REIS, 2012). Entretanto, a situação de incerteza e indefinição dos recursos financeiros para o SUS ainda permanece, devido ao descumprimento dos recursos da União e até mesmo de grande parte dos estados, acentuando o quadro de subfinanciamento da saúde e prejudicando o princípio da universalidade da atenção (MENDES; MARQUES, 2012, p. 276). 


\section{Considerações finais}

A implementação da atenção primária brasileira é um campo que precisa ser explorado sob diferentes perspectivas, em especial no âmbito da esfera federal, cuja literatura é escassa e prevalecem estudos de base municipal. Essa necessidade se justifica pela história da APS brasileira, que tem estruturação política considerada recente, mas que avançou significativamente no cenário nacional.

A ampliação em âmbito nacional da APS se deu sob forte condução federal, na figura do Ministério da Saúde, principalmente por meio da expedição de portarias e de regulamentação financeira, que refletem as decisões negociadas nas arenas decisórias do SUS. Essas negociações geralmente ocorrem numa atmosfera de intensos debates, relativos às forças de coalizão típicas do sistema presidencialista e federalista brasileiro.

O desenvolvimento da APS e de toda a política de saúde são sensíveis a essas forças, principalmente quando consideramos que a atenção primária se configura como contra-hegemônica no âmbito da política de saúde, despertando diferentes interesses, inclusive no interior da esfera federal, em que há disputa por priorização de pautas na agenda e principalmente por recursos financeiros.

Embora a APS tenha obtido ganhos consideráveis ao longo da implementação de sua política, é imprescindível sua valorização financeira e estrutural, que a possibilite ultrapassar a barreira do acesso para a totalidade da população brasileira e que seja capaz de torná-la uma sólida base para o sistema público de saúde, com condições de assumir a coordenação dos cuidados efetivamente. ${ }^{1}$

\section{Agradecimentos}

À Fundação Cearense de Apoio ao Desenvolvimento Científico e Tecnológico (FUNCAP), que financiou bolsa de mestrado para a pesquisadora.

\section{Referências}

ALMEIDA, P.F. Estratégias de coordenação dos cuidados: fortalecimento da atenção primária à saúde e integração entre níveis assistenciais em grandes centros urbanos. Tese (Doutorado em Saúde Pública) - Escola Nacional de Saúde Pública, Fiocruz, Rio de Janeiro, 2010.

ANDRADE, L.O.M.; BARRETO, I.C.H.C.; FONSECA, C.D. A estratégia de saúde da família. In: . Fundamentos e práticas da atenção primária à saúde: medicina ambulatorial. Porto Alegre: Artmed, 2006, p. 88-100. 
ANDRADE, L.O.M. et al. Atenção Primária à Saúde e Estratégia Saúde da Família. In: CAMPOS, Gastão Wagner de Sousa (org.) et al. Tratado de Saúde Coletiva. São Paulo: Hucitec, 2012. p. 845-902.

BAPTISTA, T.W.F. Análise das portarias ministeriais da saúde e reflexões sobre a condução nacional da política de saúde. Cad. Saúde Pública. Rio de Janeiro, v. 23, n. 3, p. 615-626, mar. 2007.

BRASIL. Ministério da Saúde. Atenção à Saúde. Saúde da Família. Brasília, 18 jul. 2013. Disponível em: < http://189.28.128.178/sage/> Acesso em: 18 jul. 2013.

Ministério da Saúde. Gabinete do Ministro. Portaria No 1.654, de 19 de julho de 2011. Institui, no âmbito do Sistema Único de Saúde, o Programa Nacional de Melhoria do Acesso e da Qualidade da Atenção Básica (PMAQ-AB) e o Incentivo Financeiro do PMAQAB, denominado Componente de Qualidade do Piso de Atenção Básica Variável - PAB Variável. Brasília, 19 jul. 2011. Disponível em: < http://www.brasilsus.com.br/legislacoes/ gm/108814-1654.html> Acesso em: 2011.

Ministério da Saúde. Secretaria de Atenção à Saúde. Política Nacional de Atenção Básica. Brasília: Ministério da Saúde, 2012. 108 p.

CASTRO, A.L.B. de. A condução federal da política de atenção primária à saúde no Brasil: continuidades e mudanças no período de 2003 a 2008. 2009. 215 f. Dissertação (Mestrado em Saúde Pública) - Escola Nacional de Saúde Pública Sergio Arouca, Fundação Oswaldo Cruz, Rio de Janeiro, 2009.

CASTRO, A.L.B. de; MACHADO, C.V. A política de atenção primária à saúde no Brasil: notas sobre a regulação e o financiamento federal. Cad. Saúde Pública, Rio de Janeiro, v. 26, n. 4, p. 693-705, abr. 2010.

CONILL, E.; FAUSTO, M. Análisis de la problemática de la integración de la APS en el contexto actual: causas que inciden en la fragmentación de servicios y sus efectos en la cohesión social. Rio de Janeiro: EuroSocial Salud, 2007.

FACCHINI, L.A. et al. Desempenho do PSF no Sul e no Nordeste do Brasil: avaliação institucional e epidemiológica da Atenção Básica à Saúde. Ciência \& Saúde Coletiva, v. 11, n. 3, p. 669-681, 2006.

FELISBERTO, E. et al. Análise da sustentabilidade de uma política de avaliação: o caso da atenção básica no Brasil. Cad. Saúde Pública, Rio de Janeiro, v. 26, n. 6, p. 1079-1095, jun. 2010.

FLEURY, S.; OUVERNEY, A.M. Política de saúde: uma política social. In: GIOVANELLA, L. et al. (Org.). Políticas e Sistema de Saúde no Brasil. Rio de Janeiro: Fiocruz, 2008.

HOFMARCHER, M.M.; OXLEY, H.; RUSTICELLI, E. Improved Health System Performance Through Better Care Coordination, n. 30. Paris: OECD, 2007. 
LABRA, M.E. Conselhos de Saúde: dilemas, avanços e desafios. In: LIMA, N.T (Org.). Saúde e Democracia. Rio de Janeiro: Fiocruz, 2005. p. 353-384.

LASSANCE, A. Federalismo no Brasil: trajetória institucional e alternativas para um novo patamar de construção do estado. In: LINARES, P.T.F.; MENDES, C.C.; LASSANCE, A. (Orgs.). Federalismo à brasileira: questões para discussão. v. 8. Brasília: Ipea, 2012. p. 26-35. MENDES, A.; MARQUES, R.M. Sobre a economia da saúde: campos de avanço e sua contribuição para a gestão da saúde pública no Brasil. In: CAMPOS, G.W.S. (Org.) et al. Tratado de Saúde Coletiva. São Paulo: Hucitec, 2012. p. 247-281.

NÚNEEZ, R.T.; LORENZO, I.V.; NAVERRETE, M.L. La coordinación entre niveles asistenciales: uma sistematización de sus instrumentos y medidas. Gac Sanit, v. 20, n. 6, p. 485-95, 2006.

OCKÉ-REIS, C.O. SUS: o desafio de ser único. Ri de Janeiro: Fiocruz, 2012. 180 p.

PAIM, J.S.; TEIXEIRA, C.F. Política, planejamento e gestão em saúde: balanço do estado da arte, Revista de Saúde Pública, n. 40 (n. esp.), p. 73-78, 2006.

SOLLA, J.J.S.P. et al. Mudanças recentes no financiamento federal do Sistema Único de Saúde: atenção básica à saúde. Rev. Bras. Saúde Matern. Infant., Recife, v. 7, n. 4, p. $495-$ 502, out./dez., 2007.

STARFIELD, B. Atenção primária: equilíbrio entre necessidades de saúde, serviços e tecnologia. Tradução: Fidelity Translations. Brasília: Unesco, 2002.

UGÁ, M.A.D.; MARQUES, R.M. O financiamento do SUS: trajetória, contexto e constrangimentos. In: LIMA, Nísia trindade et al. Saúde e democracia: história e perspectivas do SUS. Rio de Janeiro: Editora Fiocruz, 2005.

VIANA, A.L. et al. Mudanças significativas no processo de descentralização do sistema de saúde no Brasil. Cad. Saúde Pública, Rio de Janeiro, v. 18 (supl.), p. 139-151, 2002.

\section{Nota}

${ }^{1}$ R.M. da Silva participou da elaboração do projeto de pesquisa, da revisão bibliográfica, da pesquisa de campo, da análise e interpretação dos dados, da redação e revisão crítica do artigo. L.O.M. de Andrade participou da orientação do projeto de pesquisa, da revisão bibliográfica, da pesquisa de campo, da análise e interpretação dos dados, da revisão crítica e da aprovação da versão final do artigo. 


\section{Abstract}

\section{Coordination of Health Care In Brazil: the challenge of strengthening federal primary health care}

This study aimed to analyze the federal induction in the strengthening of primary health care (PHC) as a way to achieve coordination of care in the Brazilian health system. Quantitative and qualitative study that used documentary data, budgetary and semi-structured interviews with managers of the federal Department of Primary Care of the Ministry of Health, in years 2007-2011. Thematic content analysis and descriptive statistics were used and provided an overview of the Brazilian PHC, especially the coordination of care from the perspective of centralized induction. Strengthening of $\mathrm{PHC}$ is the basis for making care coordination in Brazil. Its prioritization is increasing within the MS, which has been conducting national policies that have generated impact in strengthening the APS and coordination of care, becoming a strong inducer of this policy, mainly through the issue of ordinances. No ordinance or specific budget has been identified for care coordination, which demonstrates the paucity of this attribute in the Brazilian PHC. Social participation was considered relevant to the scope of care coordination. The research concludes that although $\mathrm{PHC}$ has been prioritized in the agenda of the Ministry of Health, its importance does not occur homogeneously in all federal decision-making areas.

Key words: primary health care; coordination of health care; Ministry of Health. 